\title{
Pueblos Indígenas, Terrorismo y Derechos Humanos
}

\author{
Rodrigo Lillo Vera*
}

\section{El uso de la Ley antiterrorista contra mapuches}

\begin{abstract}
La ley antiterrorista chilena, № 18.314, fue dictada en el año 1984 por el régimen militar a fin de enfrentar la creciente disidencia opositora que por aquella época convergía en un único referente para desestabilizar y precipitar el fin de la dictadura. El texto de la ley fue modificado a comienzos de los noventa con el objeto de ir armonizándola con los tratados de derechos humanos, de tal manera que actualmente es totalmente diferente a la original.
\end{abstract}

Siguiendo la tendencia sentada por la doctrina internacional sobre la materia, la Ley № 18.314 no adelanta una definición de terrorismo, pero los delitos terroristas se caracterizan en la ley por incluir un elemento subjetivo en el autor: la intención de producir fundado temor en la población, o una parte de ella, de verse expuesta a delitos de la misma especie, o que se ejecute para "arrancar decisiones de la autoridad". La ley es más rigurosa que la ley común, no sólo por las altas penas que considera para los distintos delitos, sino también porque contempla una serie de medidas que agravan las situaciones del imputado y, por otra, aumentan las facultades del fiscal ${ }^{1}$. Entre estas, el uso de testigos sin rostro, las interceptaciones telefónicas, el secreto de la investigación hasta por 6 meses, fueron utilizadas por los fiscales en los casos materia de este comentario.

El 27 de julio de 2005 el Tribunal de juicio oral en lo penal de Temuco ${ }^{2}$ dictó sentencia en la causa RIT 080/2004, por la que se acusó a 8 mapuches por formar una asociación ilícita terrorista. En su parte resolutiva absuelve a los acusados Patricia Roxana Troncoso Robles, Jorge Abelino Huaiquin Antinao, Marcelo Antonio Quintrileo Contreras, Pascual Huentequeo Pichun Paillalao, Segundo Aniceto Norin Catriman y José Francisco Llanca Ahilla, todos militantes de la Coordinadora Arauco Malleco, poniendo fin al largo proceso judicial seguido en su contra que comenzó con su apresamiento en diciembre de 2002, bajo los cargos de conformar una organización terrorista ${ }^{3}$.

Si bien, no es nuevo en la historia de Chile ver sometidos a la justicia y a la represión policial a mapuches que dicen reivindicar derechos territoriales, el contexto actual contiene algunos elementos que lo caracterizan y diferencian de lo ocurrido en otros períodos.

En efecto, durante la primera mitad del siglo XX fueron frecuentes las disputas por las tierras ${ }^{4}$, caracterizadas por las protestas de los mapuches en contra de colonos y terratenientes por la usurpación de sus tierras, las
* Abogado, profesor de Derecho Indígena de la Facultad de Derecho de la Universidad Austral de Valdivia, Defensor Penal Público en la Defensoría Penal Licitada de Temuco.

1 Ampliación del plazo de detención del imputado a 10 días siendo la regla general de 24 horas sin necesidad de formalización del delito investigado; permite la reclusión en lugares especiales; mayores posibilidades de intercepción o registro de comunicaciones del imputado; restricción al régimen de visitas carcelarias; prohibición de revelación de identidad como, asimismo, que los testimonios se presten por cualquier medio idóneo que impida la identificación física formal.

2 Integrado para este efecto por el juez Aner Padilla, quien lo presidió, Ester Valencia y Héctor Hinojosa.

3 Mientras se realizaban las correcciones de este documento, el 7 de abril de 2006, el Tribunal Oral en lo

Este comentario, junto con el documento principal a que se refiere, están disponibles en www.anuariocdh.uchile.cl 
Penal de Angol dictó, en otro proceso contra mapuches bajo el marco de la ley antiterrorista, sentencia absolutoria a favor de José Osvaldo Cariqueo Saravia y Juan Antonio Colihuinca Ancaluán (RIT 21/2004). Esto se suma a la decisión no expresada del gobierno encabezado por Michelle Bachelet, de no utilizar la ley antiterrorista nuevamente por hechos de esta naturaleza, lo cual hace pensar en el fin de esta etapa, en que sólo quedarán pendientes los efectos de la aplicación de esta ley; esto es, la situación de los condenados en cumplimiento de sus penas.

4 Más recientemente, en el gobierno de transición de Patricio Aylwin se interpuso una querella por delitos de usurpación, daños y asociación ilícita (esta última dirigida principalmente contra los líderes del Consejo de Todas las Tierras que reivindicaban territorio $y$ autodeterminación), por la cual 144 mapuches fueron condenados a distintas penas. Siendo Eduardo Frei Presidente de la República, el gobierno interpuso denuncias por delitos de Ley de Seguridad del Estado a propósito de la quema parcial de camiones madereros en una ruta al interior de la Novena Región. Pero es a fines de 2001 que se inaugura esta nueva estrategia judicial para reprimir delitos cometidos por que terminaban en voluminosos expedientes judiciales. Tampoco es nueva la persecución política. De la represión sufrida por campesinos mapuches durante la dictadura militar -en su mayoría pertenecientes a asentamientos de la reforma agraria-, dan cuenta numerosos casos de detenidos desaparecidos y ejecutados políticos calificados como tales en la Comisión Nacional de Verdad y Reconciliación. Pero el uso de la ley antiterrorista, si bien no ha implicado violaciones masivas y de lesa humanidad como las de la dictadura, ha sido difundido y denunciado como nunca antes había ocurrido ${ }^{5}$. Organizaciones y organismos de Derechos Humanos, han examinado esta situación ante la posibilidad de tratarse de una persecución racial ${ }^{6}$; asimismo, distintos medios de comunicación nacionales informaron sobre hechos de violencia en el sur de Chile, e incluso la Comisión de Constitución, Legislación y Justicia del Senado evacuó un informe sobre la violencia y las demandas indígenas ${ }^{7}$.

Me refiero a proceso o etapa, porque el juicio al que se ha hecho referencia (RIT 080/2004) no es el único seguido contra mapuches en que se ha utilizado la ley antiterrorista en los últimos años. En total se trata de siete juicios, pero son sólo cuatro imputaciones de carácter terroristas: 1) incendio de casa habitación y amenazas a Juan Figueroa en la comuna de Traiguén, Novena Región; 2) incendio del fundo Poluco Podenco de propiedad de Forestal Mininco, en la comuna de Collipulli, Novena Región; 3) incendio de retroexcavadora que desarrollaba trabajos en la central hidroeléctrica Ralco en la actual comuna de Ralco, Octava Región; y 4) asociación ilícita terrorista, que estaría tras estas acciones. Dos juicios -en los cuales los imputados resultaron absueltos- fueron anulados y debieron ser repetidos por resolución de la Corte Suprema; en otros casos, los juicios no se han realizado al mismo tiempo para todos los imputados, debido a que algunos mapuches han rehuido la acción de la justicia, no presentándose ante los tribunales, alegando falta de garantías. La mayoría de estos juicios se llevaron a cabo bajo el nuevo procedimiento penal, salvo el caso Víctor Ancalaf, quien fue juzgado por hechos ocurridos en la zona de Alto Bío-Bío, Octava Región, donde a la época de la denuncia regía el sistema antiguo.

En estos juicios se ha imputado a mapuches la comisión de hechos calificados como delitos terroristas en la Ley № 18314, particularmente el incendio, las amenazas y la asociación ilícita terroristas, siendo juzgados de acuerdo al procedimiento establecido en esa norma. Alrededor de 8 mapuches han sido condenados en estos juicios, mientras que en otros se optó por su absolución.

En cuanto a las conductas perseguidas como terroristas, en la causa RIT 080-2004, por ejemplo, la fiscalía formuló acusación contra los mapuches por el delito de asociación ilícita terrorista, el cual se habría cometido por un grupo que actuó al amparo de la Coordinadora Arauco Malleco, organizándose para cometer una serie de actos ilícitos "tales como incendios y atentados contra las personas, la propiedad y el orden público" $^{\prime 8}$, los que luego difundían por internet. Todo lo anterior "con la 
finalidad de lograr el control territorial de diversos sectores de esta región [...] produciendo un temor generalizado, en orden a ser víctimas de delitos de la misma naturaleza, en personas relacionadas con las actividades agrícola y forestal en predios contiguos a comunidades indígenas intervenidas por la asociación" ${ }^{\prime \prime}$, además de alterar la paz y la seguridad.

Por otra parte, en el fallo RIT 21/2004 que absolvió a José Cariqueo y Juan Colihuinca en el proceso por delito de incendio del fundo Poluco Pidenco, el Ministerio Público consideraba que el carácter terrorista estaba demostrado por el temor justificado,

[...] ya que así lo expresó Juan Eduardo Correa Bulnes, representante de la CORMA, quien declaró que en la época en la que ocurrió este ilícito en este sector del país, en la comuna de Ercilla existía una sensación de temor de ser objeto de este tipo de ataques por parte de las empresas forestales y dio cuenta en un inserto que se publicó por su asociación relativo a más de 100 atentados ocurridos en ese periodo y que asociados le refirieron que sentían este temor [...] añadiendo que los empresarios, al igual que toda persona, tienen derecho a no vivir con miedo, en este mismo sentido están los atentados de Gerardo Cerda, mediano empresario, quien dijo que operaba con comuneros mapuches el verano del año 2001, y dio cuenta de la situación de inseguridad pública, pues sus trabajadores se vieron afectados, hubo brutales atentados en contra de los mismos, los que habitualmente eran emboscados y expulsados de los predios, situación que provocó un profundo temor ${ }^{10}$.

En ninguno de estos dos casos la sentencia fue condenatoria. En el primer caso (juicio por asociación ilícita terrorista), el tribunal decidió que la prueba aportada no alcanzaba para concluir que había una organización estructurada, que planificara la acción terrorista en la Novena Región, sino sólo que los acusados habían participado en hechos ya sancionados -como terroristas o no- por otros tribunales. Los jueces no se pronunciaron, en cambio, acerca de si estos delitos tenían o no el carácter terrorista, aunque del considerando DUODÉCIMO ${ }^{11}$ se desprende que ellos no compartían esa calificación para el caso de los mapuches, pero no quisieron contradecir sentencias anteriores dictadas por otros jueces.

Por su parte, la reciente sentencia de 7 de abril de 2006 en juicio RIT 21/ 2004 es concluyente en cuanto a que no se pudo acreditar por el Ministerio Público el carácter terrorista del incendio investigado. La sentencia consideró así que no había "antecedentes suficientes para entender que se encuentra acreditada dicha circunstancia"12. Aunque ninguno de las dos sentencias se detiene demasiado sobre la calificación, y en qué casos se puede hablar de un ilícito terrorista.

En otros dos casos, un tribunal de Angol condenó a los loncos Pascual Pichún Paillalao y Aniceto Norín Catrimán, por el delito de amenazas terroristas en contra del ex ministro Juan Figueroa Yávar (RIT 2/2003), y a 5 mapuches por el mencionado incendio del fundo forestal "Poluco Pidenco" (RIT 21/2004). En estos casos, las conductas ilícitas de los mapuches en el contexto de sus reivindicaciones territoriales.

5 En términos de vulnerarse derecho en su condición étnica.

6 En el año 2003 la Federación Internacional de Derechos Humanos (FIDH) elaboró un informe exclusivo sobre la situación de los mapuches en el sur de Chile; mientras que en el mes de abril de este año, se presentó un nuevo informe donde se hace una crítica al uso de la ley antiterrorista en contra de los mapuches. Durante el desarrollo del primer juicio de los loncos, una misión de Amnistía Internacional visitó el Tribunal y conversó con los familiares y abogados defensores de los mapuches. En octubre de 2004 Human Rights Watch, presentó junto al Observatorio de Derechos de los Pueblos Indígenas su informe los juicios antiterroristas, los tribunales militares y los mapuches en el sur de Chile. Entre el 18 y el 29 de julio de 2003, el Relator Especial de Pueblos Indígenas de las Naciones Unidas realizó una misión a Chile, formulando en su informe final una serie de recomendaciones al Estado chileno en relación con los juicios realizados en contra de mapuches.

7 Comisión de Constitución, Legislación, Justicia y Reglamento del Senado. 2003. Informe recaído en el encargo que le hiciera el 
Senado respecto del conflicto mapuche en relación con el orden público y la seguridad ciudadana en determinadas regiones. Boletín № S 680-12.

8 Considerando segundo de la sentencia de fecha 27 de julio de 2005, dictada por los jueces Aner Padilla B. Ester Valencia D. y Héctor Hinojosa A., RIT 080/2004.

9 Considerando segundo, sentencia de fecha 27 de julio de 2005, RIT 080/2004.

10 Considerando UNDÉCIMO, sentencia definitiva de fecha 07 de abril de 2006 en causa RIT 21/ 2004.

11 “...no corresponde entrar a apreciar si ellos tienen o no el carácter de terroristas; por lo demás, cabe tener presente que cualquier definición de terrorismo que se quiera enunciar debe necesariamente contener el concepto de desprecio a la vida humana, propia o ajena, concepto que no resulta del contenido de los hechos que se relataron en la audiencia". Considerando Duodécimo, sentencia RIT 080/2004.

12 Considerando DÉCIMO NOVENO de la sentencia de 7 de abril de 2006, causa RIT 21/2004.

13 En ambas sentencias, RIT 2/2003 y $21 / 2004$, el considerando referido a la calificación de terrorista es idéntico.

14 Considerando Décimo Noveno, sentencia RIT $21 / 2004$. acusados sí fueron calificadas de terroristas, utilizando idénticos $\operatorname{argumentos}^{13}$. Con el objeto de justificar esta calificación jurídica, los sentenciadores recurrieron al contexto en que ocurrieron estos hechos, señalando que:

...el ilícito establecido [...] está inserto en un proceso de recuperación de tierras del pueblo mapuche, el que se ha llevado a efecto por vías de hecho, sin respetar la institucionalidad y legalidad vigentes, recurriendo a acciones de fuerza previamente planificadas, concertadas y preparadas por grupos radicalizados que buscan crear un clima de inseguridad, inestabilidad y temor en la Provincia de Malleco, $[\ldots]^{14}$.

\section{El uso la ley antiterrorista y los Derechos Humanos de los acusados}

Aunque no existen antecedentes para vincular los delitos atribuidos a mapuches en el sur de Chile -que han sido considerados terroristas-, con los acontecimientos de Nueva York, Madrid o Londres, sí es posible afirmar que la reacción mundial generada respecto de estos últimos ha influido en los primeros. El terrorismo es hoy una preocupación mundial, desde los atentados en Estados Unidos el 11 de septiembre de 2001, los gobiernos de los países más poderosos no escatiman esfuerzos por reprimir cualquier rastro de terrorismo. Esto ha generado también una preocupación por los límites que debe tener esta lucha, emprendida por diversos Estados del primer mundo. En este sentido podemos decir que los problemas más graves que se producen en la práctica entre el combate contra el terrorismo y el respeto por los derechos humanos son básicamente dos: la tendencia de vincular toda protesta, reivindicación $\mathrm{o}$, incluso, violencia en barrios marginales y pandillas con terrorismo, y el hecho que la persecución y sanción de quienes son acusados por estos crímenes deleznables también exige la intangibilidad de los derechos humanos. Esta preocupación se manifiesta, por ejemplo, en la elaboración de un informe sobre terrorismo y derechos humanos por parte de la Relatora Especial, Sra. Kalliopi K. Koufa, que fue presentado en junio de $2004^{15}$, por mandato de la Comisión de Derechos Humanos de Naciones Unidas, así como también la presentación de otro informe sobre la materia a cargo de la Comisión Interamericana de Derechos Humanos (CIDH) en el año $2002^{16}$.

\section{Juicios antiterroristas y debido proceso}

Las amplísimas facultades que otorga la ley antiterrorista fueron ejercidas en toda su extensión por los fiscales e interpretadas de esa manera por los jueces, dando lugar a la afectación de algunos derechos de los imputados frente a la justicia en los dos casos a los que nos hemos referido. Particularmente grave es la situación provocada con la autorización del uso de testigos sin rostro, y la privación a la libertad personal en las prolongadas prisiones preventivas. 


\section{Los testigos sin rostro}

En los dos juicios seguidos contra los loncos y Patricia Troncoso en $2003^{17}$, el tribunal autorizó una singular manera de proteger a los testigos durante el juicio oral, permitiendo que éstos declarasen detrás de un biombo y con alterador de voz, de tal manera que nadie en la audiencia pudiera ver su rostro ni identificarlo. Adicionalmente, la defensa no conoció en ningún momento la identidad de los testigos, por lo que pueden ser Ilamados testigos sin rostro.

En el juicio por asociación ilícita terrorista seguida en contra de miembros de la Coordinadora Arauco Malleco, los jueces permitieron la declaración mediante el mismo método, pero en la audiencia ofrecieron a la defensa conocer la identidad de los testigos.

Si bien es cierto que los artículos 15 y siguientes de la ley sobre conductas terroristas permiten proteger a los testigos de manera incluso más intensa que la establecida en el Código Procesal Penal ${ }^{18}$, y que el artículo 18 incluye la posibilidad que el Ministerio Público presente testigos sin rostro, siempre se debe garantizar el derecho de la defensa del imputado a contra interrogar al testigo. Aunque en verdad, esta es una ecuación difícil de cumplir, pues la presencia de testigos anónimos siempre afectará de alguna manera la defensa:

[a]l negársele información sobre los nombres y los datos personales de los testigos, la defensa no puede examinar su credibilidad. Los factores relevantes pueden incluir el posible parentesco u otra relación con los acusados, las víctimas u otros testigos de la acusación; historial profesional; antecedentes penales; o detalles médicos tales como si el testigo tiene limitaciones de visión o sufre problemas de memoria. Una de las garantías más importantes contra el perjurio es la capacidad de la defensa para interrogar a los testigos sin que se restrinja su acceso a la información más pertinente ${ }^{19}$.

Más aún, cuando el Tribunal-como en estos casos-impone la prohibición de revelar su identidad a los intervinientes (fiscales, defensores, jueces, imputados y víctimas), queda vedada la posibilidad de perseguir a estos testigos en casos que incurran en falso testimonio, afectando con su declaración al imputado.

Se produce inevitablemente un conflicto entre el derecho a la vida y/o a la integridad del testigo o perito, y el derecho a defensa del imputado. Para resolver este conflicto podemos recurrir a las reglas de necesidad y proporcionalidad enunciadas por Alexy ${ }^{20}$; conforme a ellas, será posible acceder a la protección de la identidad del testigo sólo cuando se acreditare que no hay otra forma de proteger la integridad de un testigo o perito que se encuentra realmente en peligro. Por lo anterior, una actitud rigurosa de los jueces -en orden a proteger las garantías de los imputadosimplicaba en estos casos hacer este análisis y exigir a la fiscalía demostrar el riesgo que corrían los testigos y peritos, para no transformar estos mecanismos de protección (que además incluían pagos, traslados y construcción de cercos y caminos) en instrumentos que favorezcan el falso testimonio y su impunidad.
15 Koufa, Kalliopi K. 2004. "Cuestiones Concretas de Derechos Humanos: Nuevas prioridades, en particular el terrorismo y la lucha contra el terrorismo. Terrorismo y Derechos Humanos", E/CN.4/ Sub.2/2004/40, 25 de junio de 2004.

16 "Informe Sobre Terrorismo y Derechos Humanos". 2002. OEA/Ser.L/V/II.116 Doc. 5 rev. 1 corr. 22 de octubre de 2002. Original: Inglés.

17 Causa RIT 2/2003, realizado en dos oportunidades por anulación del primer juicio en el cual fueron absueltos de los cargos de incendio y amenazas terroristas.

18 El artículo 308 dispone la posibilidad del Ministerio Público y del Juez, para tomar las medidas necesarias para proteger la seguridad del testigo o perito cuya integridad pudiere verse amenazada.

${ }^{19}$ Human Rights Watch y Observatorio de Derechos Indígenas. 2004. Chile Indebido Proceso. Los juicios antiterroristas, los tribunales militares y los Mapuche en el Sur de Chile; p. 36

${ }^{20}$ Alexy, Robert, Teoría de los Derechos Fundamentales, Centro de Estudios Políticos, Madrid, 2001. 
${ }^{21}$ Caso № $11.245,1$ de marzo de 1996. Informe Anual de la Comisión Interamericana de Derechos Humanos, 1995. OEA/Ser. L./V/ II.91, Doc. 7 rev. 28 de febrero de 1996.

22 Caso Genie Lacayo, sentencia del 29 de enero de 1997.

\section{Prisión preventiva y presunción de inocencia}

En el juicio por asociación ilícita terrorista, al igual que los otros tramitados conforme a la Ley № 18.314, los imputados permanecieron en promedio un año en prisión de manera provisional, afectándose el derecho a la libertad personal y la presunción de inocencia.

La ley procesal chilena establece otros elementos adicionales al peligro de fuga que justifican la prisión preventiva. Entre estos criterios se encuentra la gravedad del delito investigado. Obviamente, la consideración de tratarse de delitos terroristas -calificación que corresponde unilateralmente al fiscal-, satisfizo los estándares de los jueces de garantías en estos casos, perdiendo la prisión preventiva su carácter excepcional. Esto genera un efecto sobre la presunción de inocencia, como lo señaló la CIDH en el caso Jiménez:

[a]umenta el riesgo de que se invierta el sentido de la presunción de inocencia cuando la detención previa al juicio es de duración no razonable. La presunción de inocencia se torna cada vez más vacía y finalmente se convierte en una burla cuando la detención previa al juicio es excesivamente prolongada dado que, a pesar de la presunción, se está privando de la libertad a una persona todavía inocente, castigo severo que legítimamente se impone a los que han sido condenados ${ }^{21}$.

Para regular esta situación, la Corte Interamericana de Derechos Humanos (Corte IDH) ha desarrollado el concepto de plazo razonable para determinar el tiempo en que puede -sin vulnerar el derecho al debido proceso- demorar la realización del juicio al imputado, especialmente si se encuentra privado de libertad. En la sentencia sobre el caso de Genie Lacayo contra el Estado de Nicaragua de 199722, la Corte IDH indicó que para determinar la razonabilidad del plazo debe atenderse a tres elementos; a saber, la complejidad del asunto, la actividad procesal del interesado y la conducta de la autoridad judicial. Adicionalmente ese tribunal tomó en consideración el análisis global del procedimiento.

Desde esta perspectiva, aunque pudiera defenderse la posición de que la investigación de un delito terrorista no puede ser breve por la complejidad que pueda revestir, esto no implica que los imputados deban estar privados de libertad durante este lapso. En este sentido, destaca el bajo estándar judicial exigido para mantener la prisión preventiva de los imputados por un tiempo tan largo, que sobrepasa el promedio de tiempo que actualmente dura la prisión preventiva en Chile.

\section{Uso abusivo de la ley antiterrorista y la estigmatización}

\section{Las conductas por las que fueron acusados los mapuches no son terroristas}

Aunque la ley antiterrorista chilena puede ser objeto de revisión en algunos aspectos (como lo amplio de los tipos descritos), los problemas advertidos en estos casos -en relación con la vigencia de los derechos humanos- no tienen que ver con la letra de la ley ni con su origen, sino 
con su interpretación y aplicación por órganos públicos. No se trata de cuestionar, por lo mismo, el hecho de que un Estado democrático cuente con instrumento legal para proteger a la población de los ataques de este tipo que vulneran los derechos humanos de manera radical.

Lo reprochable es la aplicación de la ley a hechos que no constituyen tales actos, con el objeto -no expresado- de perseguir una lucha social legítima.

Si bien no es fácil determinar qué es terrorismo, pues no existe acuerdo en el Derecho Internacional ni nacional sobre su definición, y su búsqueda permanente se ha transformado en lo que la Relatora Especial ha calificado como el "obsesionante problema de la definición: aclarar lo que es y lo que no es terrorismo" 23 , sí existen elementos que permiten identificar una conducta terrorista de una que no lo es.

La propia Relatora Especial señaló que algunas nociones sobre lo que se considera terrorismo pueden encontrarse en el Derecho Internacional Humanitario que proscribe el recurso al terrorismo ${ }^{24}$, particularmente en los Convenios de Ginebra que disponen -entre otras materias- la protección de las víctimas y la regulación de las armas usadas en conflictos armados, y que, en su artículo 3 común, según la opinión de Gasser ${ }^{25}$, "prohíbe los actos de terrorismo... sin llegar a emplear el término terrorismo" 26 .

La calificación de estos mismos crímenes como actos terroristas la encontramos no sólo en la mayoría de Convenios y Protocolos de Naciones Unidas, sino también en la jurisprudencia de tribunales internacionales. Es el caso de los autos de procesamiento del Tribunal Especial para Sierra Leona y de la sentencia de 5 de diciembre de 2003 de la Sala de Primera Instancia del Tribunal Penal Internacional para la ex Yugoslavia en el caso Galic, que condenó al acusado por el delito de terror contra la población civil como infracción las leyes o costumbres de la guerra ${ }^{27}$.

En contraste, los delitos por los que los mapuches han sido perseguidos y/o condenados afectan la propiedad, y sólo indirectamente la vida humana (como el repeler a guardias forestales que intentan apagar un incendio, amenazas a propietarios agrícolas, o enfrentarse con palos y piedras con la policía). Si bien son ilícitos, no admiten ninguna comparación con los atroces crímenes regulados en el Derecho Internacional Humanitario, por más amplio que se considere el terrorismo, ni graves que nos parezcan los delitos cometidos.

\section{Lucha antiterrorista y democracia}

Ahora bien, las consecuencias de una aplicación extendida de la ley antiterrorista no son sólo de carácter penal ni su vulneración se vincula exclusivamente a la del principio de legalidad, sino que, además, significa, en la práctica que se está -mediante el juzgamiento como terrorista de una conducta que no lo es- estigmatizando una lucha legítima, como lo es la reivindicación territorial de un pueblo indígena. Al calificar estas
${ }^{23}$ Koufa, Kalliopi K., op. cit., párr. 12. De hecho, la Convención Internacional para la Represión de la Financiación del Terrorismo es la única que ha definido el terrorismo en su artículo 2(1) (a) y (b).

${ }^{24}$ Koufa, Kalliopi K., op. cit., párr. 15

25 Gasser, H. P. "Acts of terror, terrorism and internacional humanitarian law", en Internacional Review of the Red Cross, 2002, vol. 84, № 847, citado por Koufa, Kalliopi K. 2004; op. cit., párr. 19.

${ }^{26}$ Este artículo -idéntico en los cuatro Convenios de Ginebraprohíbe respecto de las personas que no participen en hostilidades, los atentados contra la vida y la integridad física, las mutilaciones, los tratos crueles e inhumanos, la tortura y los suplicios, la toma de rehenes, atentados contra la dignidad personal, condenas ejecutadas y sentencias dictadas sin previo juicio con garantías judiciales, etc.

27 Koufa, Kalliopi K., op. cit., párr. 25. 
28 Stavenhagen, Rodolfo, "Derechos Humanos y cuestiones indígenas. Informe del Relator Especial sobre la situación de los derechos humanos $y$ las libertades fundamentales de los indígenas", presentado de conformidad con la resolución 2001/57 de la Comisión. Adición MISIÓN A CHILE". E/ CN. 4/2004/80/Add.3, 17 de noviembre de 2003; párrs. 69 y 70. conductas como terroristas, en definitiva se incurre en la cuestionada perspectiva de entender que un movimiento social implica terrorismo, transformando los problemas sociales en problemas de seguridad, con las consiguientes consecuencias para la democracia.

Esto es lo que advirtió precisamente el Relator Especial de Pueblos Indígenas de las Naciones Unidas en su informe sobre la misión a Chile, realizada entre el 18 y el 29 de julio de 2003. Entre las recomendaciones que sugiere al Estado chileno, en base a lo que fueron sus observaciones, Stavenhagen formula una especie de advertencia:

69. "Bajo ninguna circunstancia deberán ser criminalizadas o penalizadas las legítimas actividades de protesta o demanda social de las organizaciones y comunidades indígenas. 70. No deberán aplicarse acusaciones de delitos tomados de otros contextos ("amenaza terrorista", "asociación delictuosa") a hechos relacionados con la lucha social por la tierra y los legítimos reclamos indígenas ${ }^{\prime 28}$.

Considerando los efectos que, en todo caso, han producido estos hechos en los imputados mapuches, el Relator recomendó:

"71. [...] que sea revisado el caso de los lonkos (sic) procesados, Pascual Pichún de Temulemu y Aniceto Norín de Didaico (IX Región de la Araucanía), con estricto apego a las garantías del debido proceso establecidas en las normas internacionales de Derechos Humanos"; y "75. [...] que el Gobierno de Chile considere la posibilidad de declarar una amnistía general para los defensores indígenas de los derechos humanos procesados por realizar actividades sociales y/o políticas en el marco de la defensa de las tierras indígenas". 